\title{
Las características de las TIC en el inicio de la grafoescritura.
}

\section{Tomás Cámara Pastor}

Maestro especialista en pedagogía terapéutica (La Rioja). camarapastortomas@gmail.com

\begin{abstract}
\$EWBDFW

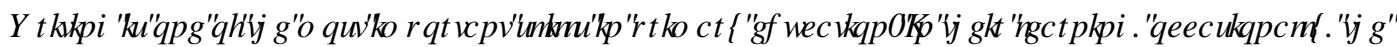

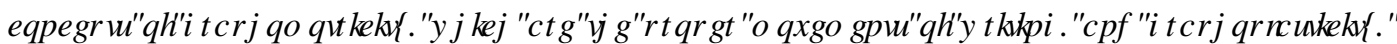

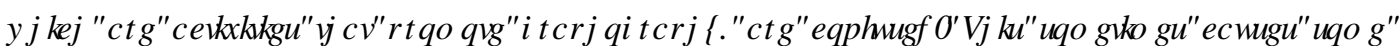

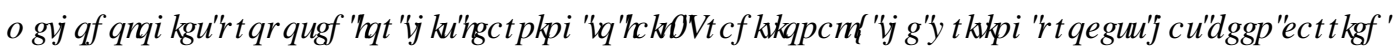

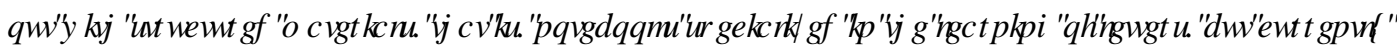

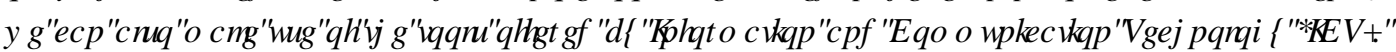

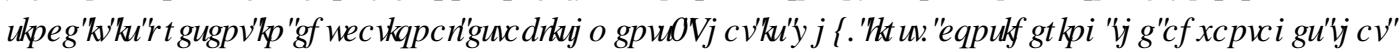

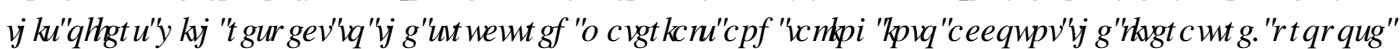

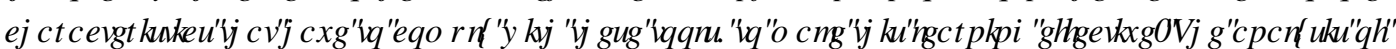

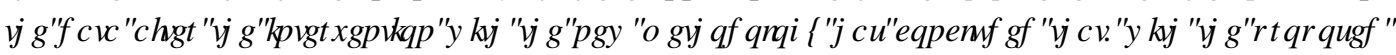

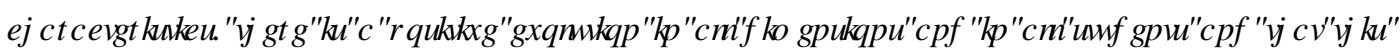

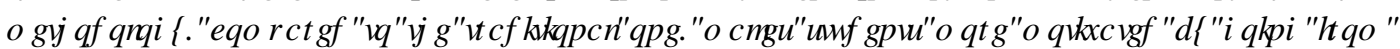
HSHQMYHZ RLNUKLEHQJDDJDP HD
\end{abstract}

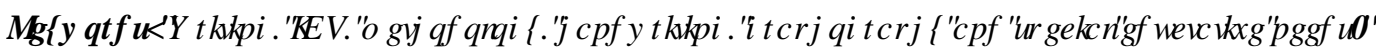

\section{HXP HQ]}

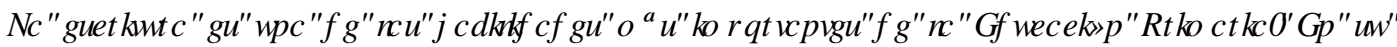

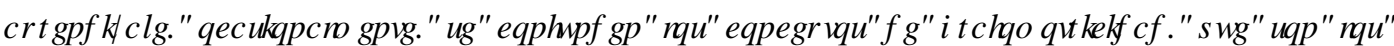

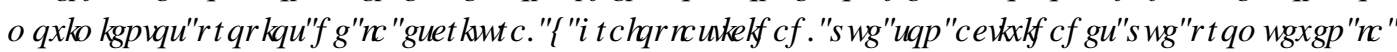

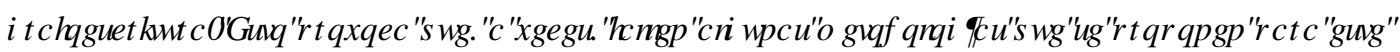

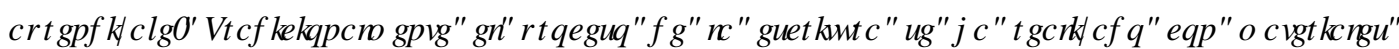

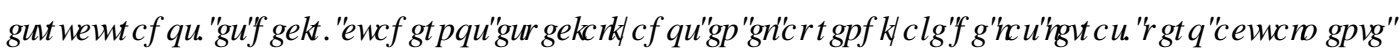

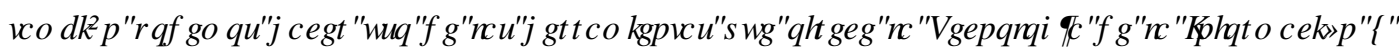

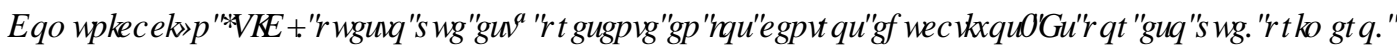

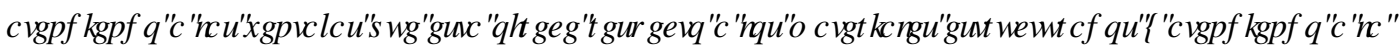

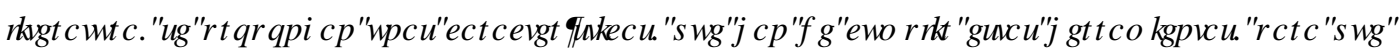

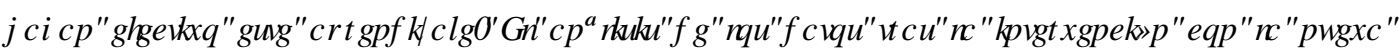

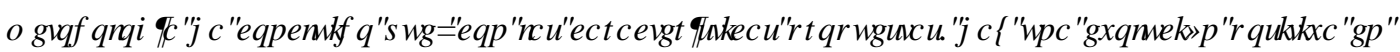

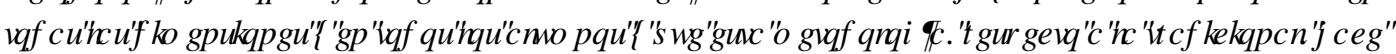

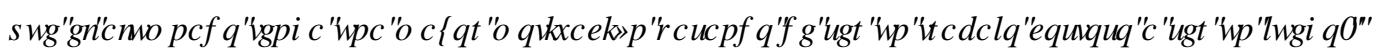

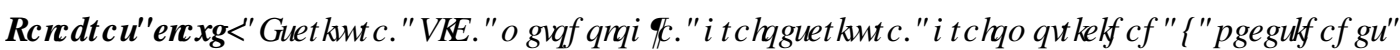
HEXFWYDWHSH FWOAW 


\section{Introducción}

\subsection{Cuestiones Previas.}

La Tecnología de la Información y Comunicación (TIC) actualmente pertenece a todos los planos de la vida, incluso, desde hace ya algún tiempo lo podemos ver en el campo de la educación. El uso que se hace de ellas es un tema en continuo cambio; por sus avances, por lo que la investigación de esta área es un tema actual. La escritura es una habilidad clave en el proceso educativo por lo que el uso de las TIC, como elemento para la gamificación de este contenido, no se puede obviar sin una previa reflexión de las características que deben cumplir para que realmente sea efectivo y de calidad el aprendizaje en el que se inmiscuye al alumnado.

Aprender a leer y a escribir (alfabetización) es uno de los elementos fundamentales en la educación infantil. Pero, por otro lado, esta etapa no es una educación obligatoria por lo que muchos niños y niñas que no han asistido no tienen porqué haberse iniciado en el proceso lector y de escritura en el paso a la educación primaria. Es decir, en los cursos de primero y segundo. El aprender a escribir es uno de los ejes vertebrales que rigen el acceso y expresión del conocimiento, de ahí la importancia de que este aprendizaje se lleve adecuadamente con el fin de culminar con una escritura competente que le ayude transversalmente a adquirir el aprendizaje, no solo de las asignaturas relacionadas con la lengua, sino de todas las demás que están presentes en el currículo. El proceso por el que enseñamos a escribir, aunque suele estar en un segundo plano por la importancia del significado de lo que se escribe, es gradual y debe ser coordinado con la maduración neuromotora, pues no se aprende, si no hay una preparación antes de iniciar la escritura (Portellano, 2002). Por lo tanto, desde el inicio del aprendizaje este se va a ver influido por el desarrollo de cada uno de los discentes pudiendo ser incluso que este presente diferentes cualidades no recogidas como típicas, es decir, con diversidad funcional, que pueden afectar al proceso. Es el ejemplo de personas que no tienen un desarrollo madurativo adecuado a su edad, que presentan diferentes trastornos relacionados con el tono muscular, la memoria, el razonamiento, sin olvidarnos de la visión, pues sin un correcto funcionamiento de los ojos tampoco es viable la escritura, entre otros muchos.

Este trabajo pretende buscar, entre todas las herramientas y conocimientos sobre el proceso de aprendizaje de la escritura, un marco de referencia y común en el trabajo de la grafomotricidad con el uso de herramientas tecnológicas que hoy en día están al alcance de la mayoría de los centros educativos. Ya que de no ser así se puede llegar a realizar una mala $S W[L V$ de las herramientas tecnológicas y, por tanto, no conseguir los objetivos propuestos. Sería entonces culpa, no del alumnado ni de las herramientas, por no responder como se espera, sino del docente por no saber emplearlas adecuadamente.

\subsection{Agentes Implicados en la Grafomotricidad.}

Los dos aspectos fundamentales que los docentes deben entender en el proceso de escritura son, por un lado, la motricidad fina definida como la capacidad de organizar y ordenar acciones motrices que tienen un fin y que además deben de ser ejecutadas con precisión, eficacia, economía y armonía, por lo que para realizar estas acciones con acierto debe estar implicado todo el sistema nervioso (Rodríguez, 2010). Este aspecto va relacionado con el hecho de los movimientos de muñeca y dedos entre los que se encuentra, entre otros de gran importancia para la escritura, la pinza digital que es imprescindible (Alsina, 2010). Por otro lado, la maduración y preparación cognitiva para poder realizar con éxito los trazos de la escritura. Todo ello siempre y cuando no haya factores que retrasen el proceso. En conclusión, la noción que se tiene de la escritura se tiene que concebir, a partir de la teoría Vigostkyana, como un aprendizaje de varios factores (neuropsicológicos, motrices, intelectuales...) que han de desarrollarse con el discente para intentar desarrollar cada vez un poco más a través de su zona próxima de desarrollo. Es decir, no es un proceso repetitivo en el que en algún momento se espera que el discente pase del lenguaje oral al escrito. A menudo, 
dos de los factores que más fallan en este proceso son la escuela y la familia pues no comprenden la escritura como proceso, sino como una habilidad que simplemente, por edad, aparece (Cassany, 2002).

\subsection{La Grafomotricidad y la Grafoplasticidad. Aclaración de Conceptos.}

Normalmente en el aprendizaje de la escritura encontramos dos tipos de actividades y a menudo son confundidas o no son bien delimitadas, aunque tienen como base común el producir experiencias y estímulos al niño para conseguir competencias en el lenguaje escrito.

Por un lado, se contempla la grafoplasticidad. Esta se puede definir por aquellas actividades que proponen los docentes para que las destrezas del niño mejoren y puedan aprender otras nuevas que les ayuden a continuar con su proceso de aprendizaje (Guangasi-Yancha, 2015). Otros autores aportan a esta definición que estas actividades son artísticas con intención comunicativa que involucra en los niños y niñas el desarrollo de los sentidos, la sensibilidad, percepción del medio y el desarrollo de la motricidad que permite e incide en la lectoescritura (Males-Vasco y Merino-Suing, 2010). Estas técnicas y estrategias son, entre otras, collages, moldear, pintar y recortar, muy utilizadas en las primeras etapas de la educación. Todas estas se deben aplicar con los recursos apropiados para ello y, además, las actividades deben ser propuestas en tareas que promuevan que el niño sea un agente activo con el fin de que el aprendizaje sea significativo (Montessori, 2004). Hay que aclarar que para realizar este tipo de actividades no son necesarios materiales estructurados (creados específicamente para ello), sino utilizar materiales no estructurados (no creados para ello) (Montilla, 2015). Es decir, los inconvenientes de los materiales estructurados son: por un lado, su precio, ya que son de coste elevado. Por otro lado, al ser de uso individual, su vida es limitada, pues concluye con un uso. Es por eso que se recomienda el uso de materiales no estructurados, son baratos, cotidianos, sencillos de conseguir (monedas, pinzas, arroz, etc.), accesibles a todo el alumnado y algunos de ellos se puede utilizar un número ilimitado de veces.

Por otro lado, está la grafomotricidad que son los movimientos de la escritura (Suárez, 2002). Las competencias relacionadas con la grafomotricidad son: a) la direccionalidad, que es una característica, con dos factores influyentes, lo visual y lo motor, que se debe automatizar y que debe ser en barrido de arriba a abajo y de izquierda a derecha, b) la pulsión tónica, que es la capacidad de presión, de forma consciente, del lápiz u otro instrumento sobre el papel, de forma que la escritura no se vea por exceso ni por defecto, c) la coordinación visomotora, que es la capacidad de orientar el trazo atendiendo a las necesidades de la escritura y d) la descodificación perceptivo motriz, que se refiere a la escritura como un canal por el que trasladamos lo que escuchamos o vemos a escritura. Además, cabe destacar en todos los procesos de forma subyacente el papel de la memoria como un factor indispensable para representar e interpretar un código (García, 2002).

En conclusión, en las escuelas se utilizan dos tipos de actividades. Por un lado, las grafoplásticas que son aquellas que ayudan y proporcionan las herramientas y destrezas que preparan para el acto de escribir y, por otro, las actividades grafomotrices que son aquellas propias de la escritura. Por lo tanto hay que saber diferenciar cada una de ellas, ya que no podemos decir que trabajamos la grafomotricidad sin los elementos indispensables de la escritura.

\subsection{Las Tecnologías de la Información y Comunicación en el Aprendizaje}

En educación existen numerosos recursos didácticos. Cuando se utilicen, deben atender a unas necesidades educativas como son: la aproximación o facilitación de los contenidos, aumentar las experiencias de aprendizaje, desarrollar habilidades cognitivas, innovación en las metodologías y aumentar las posibilidades de la evaluación (Blázquez y Lucero, 2002). Además, estos recursos, en términos tecnológicos, se pueden clasificar en recursos de información, recursos de colaboración y recursos de 
aprendizaje (Véase Figura 1), aunque haya recursos que según su uso o intención pueden ser clasificados en varios (Cacheiro, 2011).

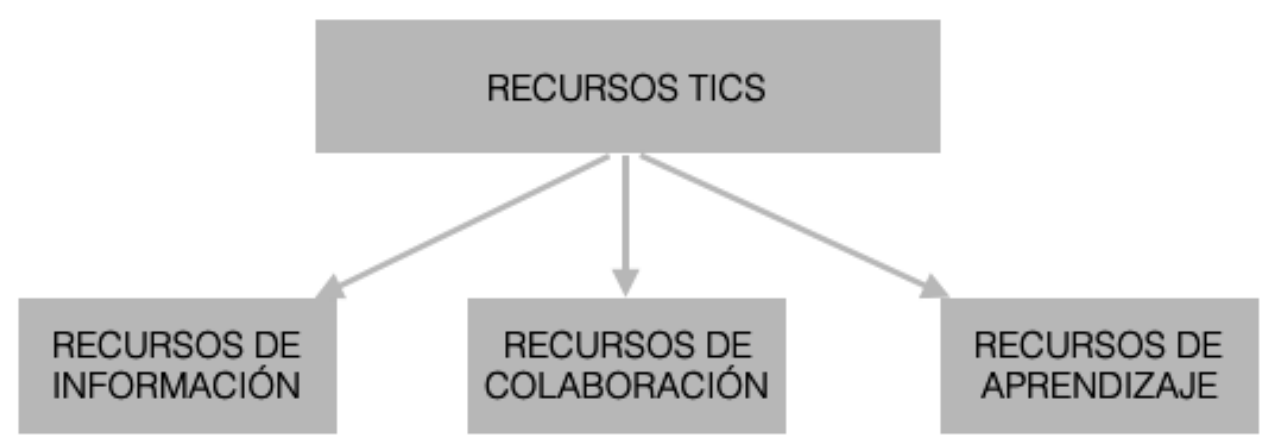

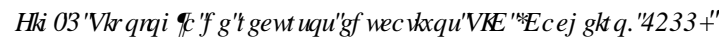

Los recursos de información son aquellos que nos ofrecen datos o información sobre un tema por ejemplo: las webgrafías, enciclopedias, documentales, etc. (Cacheiro, 2011). Hay que tener especial cuidado con estos recursos pues hoy en día hay una sobreinformación accesible al alumnado que no siempre es correcta (Medina, 2009). Por otro lado, los recursos de la colaboración son aquellos que permiten que varias personas puedan trabajar en un mismo proyecto por ejemplo: wikis, blog, etc. Estos son muy beneficiosos por su ruptura con el trabajo individual, el aislamiento académico y por el fomento del trabajo en equipo, adopción de roles, liderazgo, etc. (López y Lorenzo, 2008). Por último, los recursos del aprendizaje son aquellos que permiten la adquisición de conocimientos por ejemplo: recursos educativos, tutoriales, SRGDWMH/RRM-letc

El uso de las TIC como herramientas en el aprendizaje son valiosas porque poseen unas características innovadoras para la educación como por ejemplo que se puede aprender tanto IQ $U W$ como de manera $R Q D Q H$ los datos pueden ser almacenados y recuperados en cualquier momento para ser analizados e intervenir, la información puede ser unidireccional o bidereccional, incrementa la posibilidades de acceso e individualización de la educación y todo ello para poder crear contextos de aprendizaje más eficaces, activos, cómodos y motivantes (Casado, et al., 2007).

\section{Objetivos}

El objetivo de este trabajo es triple:

a) Exponer un marco teórico entorno al aprendizaje de la escritura.

b) Proponer unas características que deban cumplir las herramientas tecnológicas en el proceso del aprendizaje de la escritura.

c) Analizar la mejora con la metodología y las características propuestas.

\section{Desarrollo de la innovación}

\subsection{Las TIC en el Proceso de Enseñanza-Aprendizaje de la escritura.}

Una vez conocidas la clasificación y las características de las TIC, en cuanto a su importancia en educación, y la diferencia entre las actividades grafoplásticas y las grafomotrices se va a proceder a proponer diversas características para utilizar diferentes aplicaciones y herramientas tecnológicas para ser utilizadas con beneficio en proceso del aprendizaje de la escritura. 
Por un lado, la grafoplasticidad se propone que el proceso de enseñanza-aprendizaje sea sin herramientas tecnológicas, ya que hay que mejorar el tono muscular, etc; Aunque sí se pueden utilizar para cosas concretas, ha de ser el docente quien según su criterio decida el método más efectivo para su grupo-clase. Por otro lado, en la grafomotricidad sí se pueden introducir herramientas tecnológicas como son las aplicaciones que se enmarcan en el aprendizaje de los trazos de las letras del abecedario. Pero atendiendo a aquellas cualidades que son propias de la grafomotricidad se propone una serie de características para que lo que se trabaje sea este concepto y no la grafoplasticidad.

Se propone que una de las características que las aplicaciones deben cumplir, pero no solo en este caso, sino en todos en los que se utilice la tecnología, es que lo que se haga no sea sustituido por elementos físicos en los que se pueda hacer manipulando, otra de las características que se propone es que los trazos se realicen con un lápiz para pantallas ya que sino no serían movimientos propios de la escritura, como expone la definición de grafomotricidad y por tanto estaríamos trabajando grafoplasticidad. Además, otra característica que se propone es que la aplicación no debe ser una pizarra con un fondo guía donde puedas marcar trazos por cualquier sitio de la pantalla, pues de ser así no hay una regulación de por dónde debe ser el trazo (Véase figura 2).

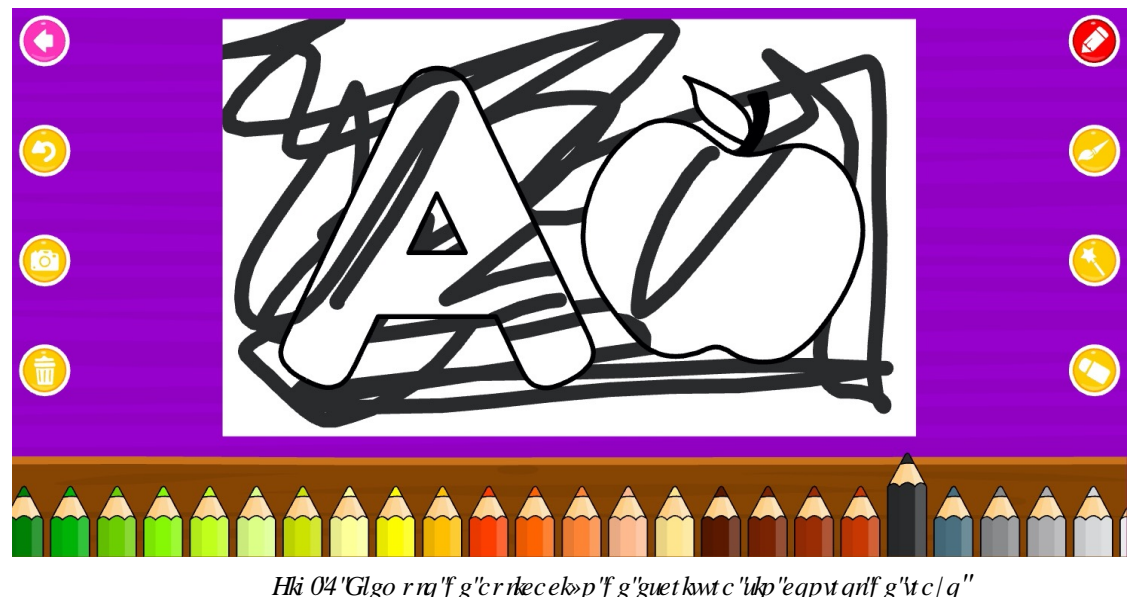

Como se puede observar, al no haber ninguna guía ni autorregulación del aprendizaje no estamos trabajando la grafomotricidad, la aleatoriedad no permite un aprendizaje de los trazos y por tanto no tiene sentido ni hacerlo en un herramienta tecnológica, pues se podría hacer lo mismo con papel ni porque realmente no se está aprendiendo los pasos y trazos de la grafías de las diferentes letras. Además, y en concordancia con este punto, en este caso lo correcto debe ser que la aplicación solo te deje pintar por el lugar del trazo de la letra y te dé indicaciones de la secuenciación del trazo (véase Figura 3).

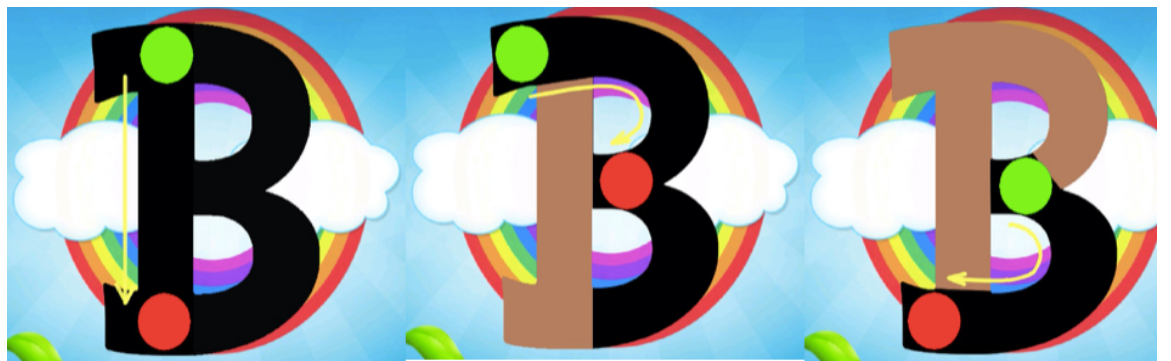

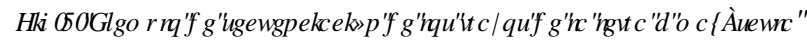

Otra de las características que se propone que cumplan las aplicaciones es que con cada letra esté relacionado, primero el fonema y luego, además, sea utilizado en una palabra para poder recordarla mejor. Por último, y aunque parezca obvio, se debe tener en cuenta que el abecedario que se utilice esté en el 
idioma que queremos trabajar, pues en este caso el español presenta la letra " $\tilde{n}$ " cuyo fonema es $/ \mathrm{n} / \mathrm{y}$ fonemas como la /ĉ/ y la / $1 /$ ( corresponden a los grafemas "ch" y "ll" respectivamente) que también se deben trabajar y que solo son propios de este idioma. Asimismo, no será conveniente en otros idiomas porque, aunque se compartan letras, no se comparten los fonemas y a la hora de hacer una copia de los fonemas que le llegan por el canal auditivo y plasmarlos en papel puede que les dificulte por no saber el grafema al que se corresponde el fonema.

\subsection{Participantes.}

La muestra ha sido intencional, ya que los participantes son alumnado con necesidades educativas especiales (ACNEE), que por diversas razones todavía no han adquirido correctamente la escritura. La muestra final consta de 15 participantes de los cuales 12 son varones $(80 \%)$ y 3 mujeres $(20 \%)$, de edades comprendidas entre los 6 y los 8 años $(M=7,13)$.

\subsection{Evaluación del Progreso.}

Para poder evaluar de forma objetiva la evolución de estos participantes, se procede a realizar un instrumento DGKRRWEste es\una rúbrica de competencias adquiridas del proceso de la escritura con una escala Likert. La escala Likert, es una herramienta utilizada por el ámbito de la psicología para medir, de una forma escalada, un conjunto de estímulos, respuestas, etc. de manera que se puede cuantificar las respuestas de manera cuantitativa (algo, poco, nada...) o la frecuencia con la que suceden (siempre, a veces, nunca...) (Likert, 1932). La que la investigación va a utilizar va del 1-5 entendiendo 1 como nada adquirida la competencia y 5 totalmente adquirida la competencia pudiendo así tener 2 valores extremos 2 intermedios y un punto medio. La temporalización de la evaluación se hará al inicio de la investigación y otra al cabo de 3 meses para comprobar en qué medida han mejorado y en qué aspectos. Además, también hay que contar con una evaluación por parte de las familias, con el fin de conocer en qué grado motiva al alumnado estas herramientas frente a las propuestas anteriormente, ya que aparte de hacer estas actividades en el aula, se propone que durante los fines de semana también se practique en el domicilio. Esta dimensión será evaluada con una pregunta con una escala likert del 1-5, que corresponde a: 1 nada motivado y 5 muy motivado.

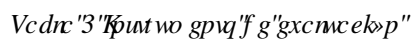

\begin{tabular}{|c|c|c|c|c|c|}
\hline Dimensiones & 1 & 2 & 3 & 4 & 5 \\
\hline Trazo & $\begin{array}{c}\text { El trazo no se } \\
\text { ajusta a las letras }\end{array}$ & $\begin{array}{c}\text { El trazo no se } \\
\text { ajusta, en su gran } \\
\text { mayoría, a las } \\
\text { letras }\end{array}$ & $\begin{array}{c}\text { El trazo se ajusta, } \\
\text { en algunas, a las } \\
\text { letras }\end{array}$ & $\begin{array}{c}\text { El trazo se ajusta, } \\
\text { en su gran } \\
\text { mayoría, a las } \\
\text { letras }\end{array}$ & $\begin{array}{c}\text { El trazo se ajusta a } \\
\text { las letras }\end{array}$ \\
\hline $\begin{array}{c}\text { Dirección y } \\
\text { sentido }\end{array}$ & $\begin{array}{l}\text { La dirección y } \\
\text { sentido no se } \\
\text { ajusta a las letras }\end{array}$ & $\begin{array}{c}\text { La dirección y } \\
\text { sentido, en su gran } \\
\text { mayoría, no se } \\
\text { ajusta a las letras }\end{array}$ & $\begin{array}{c}\text { La dirección y } \\
\text { sentido, en } \\
\text { algunas, se ajusta a } \\
\text { las letras }\end{array}$ & $\begin{array}{c}\text { La dirección y } \\
\text { sentido, en su gran } \\
\text { mayoría, se ajusta } \\
\text { a las letras }\end{array}$ & $\begin{array}{c}\text { La dirección y } \\
\text { sentido se ajusta a } \\
\text { las letras }\end{array}$ \\
\hline Dictado & $\begin{array}{c}\text { No escribe el } \\
\text { fonema dictado o } \\
\text { lo hace de manera } \\
\text { incorrecta }\end{array}$ & $\begin{array}{c}\text { La mayoría de } \\
\text { fonemas dictados } \\
\text { no los escribe o lo } \\
\text { hace de manera } \\
\text { incorrecta }\end{array}$ & $\begin{array}{l}\text { Algunos fonemas } \\
\text { dictados los } \\
\text { escribe de manera } \\
\text { correcta }\end{array}$ & $\begin{array}{l}\text { La mayoría de los } \\
\text { fonemas dictados } \\
\text { los escribe de } \\
\text { manera correcta }\end{array}$ & $\begin{array}{l}\text { Todos los fonemas } \\
\text { dictados los } \\
\text { escribe de manera } \\
\text { correcta }\end{array}$ \\
\hline Motivación & $\begin{array}{l}\text { Está nada } \\
\text { motivado }\end{array}$ & $\begin{array}{c}\text { Está muy poco } \\
\text { motivado }\end{array}$ & Está algo motivado & Está motivado & $\begin{array}{l}\text { Está muy } \\
\text { motivado }\end{array}$ \\
\hline
\end{tabular}




\section{Resultados}

Los resultados que se han obtenido no han sido tratados de manera global, sino particularizando en cada una de las dimensiones. En azul, se ve en qué punto de la escala Likert se encontraban este alumnado al inicio de la intervención y en rojo al final de la misma. Las tablas representan en el eje $\mathrm{x}$ el valor de la escala Likert en la que se encuentran, mientras que el eje y muestra el tanto por ciento del alumnado que se encuentra en la misma. A continuación se detallará cada dimensión que se pretendía evaluar por separado.

Por un lado, la dimensión "trazo" al inicio de la evaluación el 100\% del alumnado se encontraba entre los valores $1(73,4 \%)$ y $2(26,6 \%)$, una vez transcurridos los tres meses la gran mayoría de los alumnos habían llegado al valor $4(86,7 \%)$ y 2 de ellos se encontraban en el valor 3 (13,3\%) (ver Fig. 4).

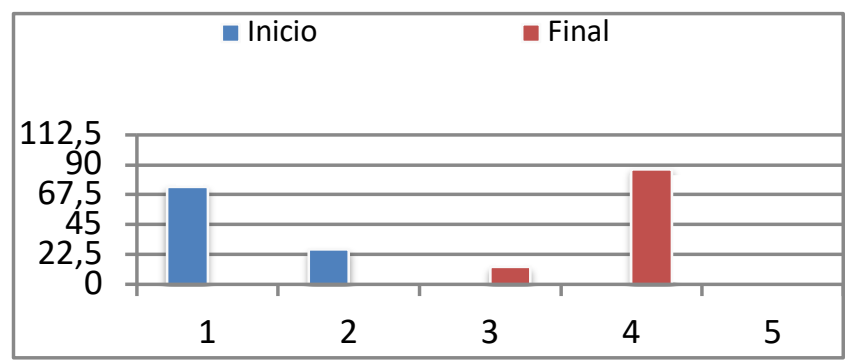

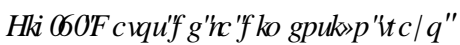

Por otro lado en la dimensión "dirección y sentido" al inicio de la investigación el valor predominante era el $1(93,3 \%)$ y solo un participante se encontraba en el valor $2(6,7 \%)$, en la evaluación final el valor predominante era el $4(86,7 \%)$, un alumno se encontró en el valor $5(6,7 \%)$ y otro en el valor $3(6,7 \%)$ (ver Fig. 5)

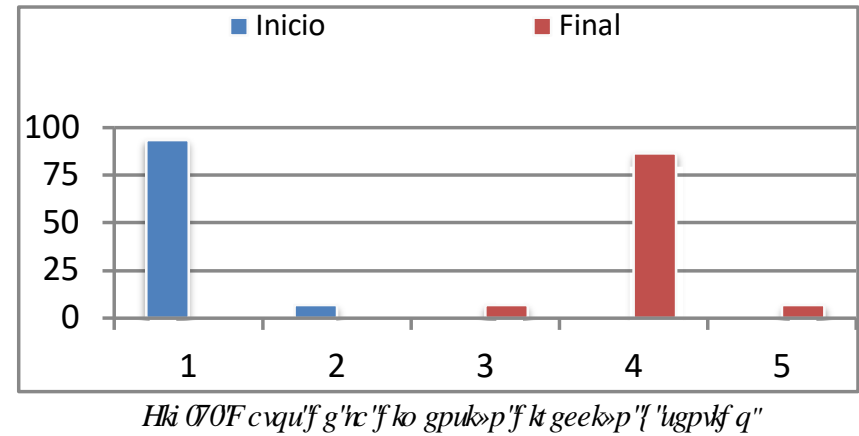

En la dimensión "dictado" el 100\% del alumnado se encontraba en el valor 1 al inicio de la investigación, al final de la misma se consiguió en que un 10 de ellos (66,6\%) estuvieran en el valor 3, 3 de ellos en el valor $2(20 \%)$ y 2 en el valor 4 (13,3\%) (ver Fig. 6).

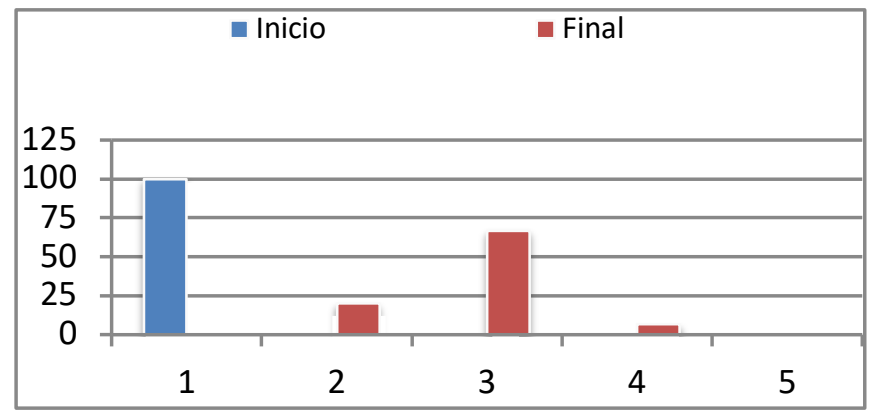

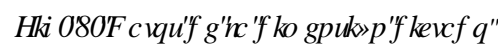

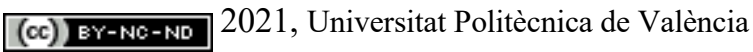

CRQJUHRT, Q5 HGHपिए। 
Por último, la dimensión "motivación" ha sido muy relevante en esta investigación ya que el del alumnado se encontraba en los valores $1(80 \%)$ y $2(20 \%)$ mientras que con esta nueva metodología al final de la investigación se invirtieron los resultados un $80 \%$ se encontraba en el valor 5 y un $20 \%$ se encontraba en los valor 4 (ver Fig. 7).

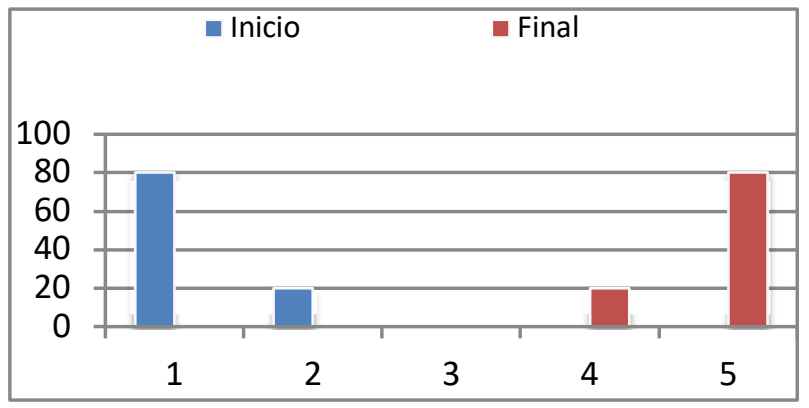

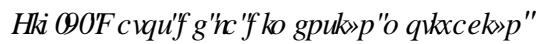

\section{Conclusiones}

Como se ha corroborado, la metodología, con las características propuestas, han funcionado y se ha mejorado la escritura en este grupo de alumnos. Uno de los datos de mayor relevancia es que al final de la investigación, todos ellos habían aprendido correctamente los trazos y dirección y sentido de las letras. Por otro lado, la dimensión "dictado" es una de las más complejas porque no solo implica la reproducción de las letras sino también una conciencia fonológica. Es por eso que, aunque no se han conseguido tanta mejoría en comparación con otras dimensiones, se puede remarcar que todos han mejorado desde el inicio de la misma. Por último, cabe destacar la dimensión de la motivación, siendo una de las que más se ha impulsado ya que escribir ha pasado de ser algo tedioso a un juego, muchas familias, ya que han sido ellas quienes han evaluado esta dimensión. Por un lado, han visto una evolución mucho mayor que en otros años con otras metodologías, por otro lado señalaban que sus hijos han pasado de no querer o tener problemas con ellos para hacer la tarea relacionada con la escritura a querer hacerla y divertirse. También, como aspecto negativo, se señala que en algunos casos han surgido conflictos cuando se pedía la finalización de la actividad y a la hora de controlar el tiempo de juego, ya que muchos de ellos no querían dejar de hacerlo para atender otras cuestiones. A estos datos, con el trabajo de esta metodología, hay que añadir otras variantes que han hecho que se produzca esta mejora, como son; el trabajo de docentes especialistas en audición y lenguaje en el centro además de otros profesionales externos al centro como logopedas, psicólogos, etc.

Aprender a escribir, valga la redundancia, se hace escribiendo, pero hoy en día hay muchas formas de escribir y muchas maneras de enseñar a hacerlo, mejores o peores, cada docente se debe adaptar al contexto y al discente en cuestión para darle la mejor posibilidad en la que él aprenda. Una de las formas, entre otras muchas, y dada la entrada tecnológica a la vida del alumnado y en las aulas, es con las TIC, pero sin antes hacer una reflexión sobre aquellas herramientas que se utilizan para el aprendizaje de la escritura, con el fin de que el docente sea consciente en todo momento de lo que se está trabajando con cada tipo de actividad. En lo que concierne a la grafomotricidad hay grandes confusiones con la grafoplasticidad y es por eso que se deben tener en cuenta las características que deben cumplir las TIC mencionadas anteriormente. Por otro lado, hay que tener en cuenta que estos ejercicios son unos posibles entre otros muchos, que todos pueden ser muy buenos para el aprendizaje del proceso de la escritura. Estos ejercicios en concreto, utilizando la tecnología como punto de partida, se puede repetir cuantas veces se quiera el movimiento de la letra, ya que no es un material tangible y finito como pueden ser las plantillas de caligrafía en las que vienen las diferentes formaciones de las letras secuenciadas por pasos desde las primeras que tienen varias indicaciones o ayudas hasta las últimas donde es total autonomía, pero que si una vez que el 
alumnado ha terminado con ellas y no las ha entendido o no es capaz de reproducirlas no puede hacer más. Además, con el uso de las TIC se está utilizando la gamificación como método para enseñar. Esta metodología motiva al niño a no cansarse y fatigarse demasiado pronto con este tipo de ejercicios, que normalmente suelen ser repetitivos y en algunos casos llegan a aburrir, por lo que se pierde la atención y el interés. Por último, y en relación con el aburrimiento, este tipo de herramientas individualiza el aprendizaje pudiendo atender a las necesidades de diferentes ritmos de aprendizaje haciendo que quienes tengan la capacidad puedan avanzar más rápido y no se aburran y se les permita dedicar tiempo a otras tareas y enriquecer el currículo mientras que a las personas que más les pueda costar les da un aliciente de refuerzo y motivación que haga que no abandonen tan rápido y se mantengan más tiempo realizando la tarea.

Cabe destacar que estas herramientas pueden ayudar al alumnado con necesidades educativas específicas de apoyo educativo (ACNEAE) al aprendizaje de la grafía de las letras. Pudiendo ser algunos de estos casos, sin tener que especificar en ninguno, pues no hay un ACNEAE en concreto al que le pueda servir más que a otros, pero sí que se propone que esta enseñanza de la grafoescritura a partir de la tecnología sea utilizada para aquellos que necesiten de mucha repetición de una misma tarea para la adquisición del contenido o de la habilidad. Esto es debido a que gracias a este tipo de herramientas la información va acompañada de un apoyo que puede resultar valioso para resaltar y apoyar la formación, como pueden ser pictogramas, elementos de la lectura fácil etc. Además, también pueden cumplir una función de estructuración de los contenidos o procesos metodológicos de enseñanza-aprendizaje, es decir, guiar al alumnado que se encuentra perdido en el conjunto del conocimiento hacia donde debe ir y secuenciar su conocimiento.

Como conclusión, este trabajo ha pretendido aportar unas directrices y características que deben de tener las aplicaciones que nos ofrecen las diferentes tecnologías que se encuentran actualmente a disposición de cualquier centro educativo en el proceso del aprendizaje de la escritura, que en ocasiones se hace tan arduo y duro tanto para el alumnado, porque no llega a alcanzar los contenidos y se ve respecto a sus compañeros atrasado; como para las familias, porque los refuerzos no aportan los resultados requeridos o esperados por diferentes causas, falta de tiempo, falta de conocimientos, etc.- y, por último, para los profesores que no entienden cómo no avanza el aprendizaje a pesar de darle todos los recursos a su disposición y por tanto no consigue que el grupo-calse avance de manera óptima. Todo ello puede crear una frustración de todos los agentes implicados en el aprendizaje del niño que al final le afecte a nivel psicológico y emocional creando un rechazo a las actividades, a la lectura y escritura o en un caso extremo a la propia institución. Así pues, se propone que una vía de trabajo pueda ser con este tipo de herramientas, pudiendo atender a metodologías más innovadoras, individualizas y motivadoras.

Por último, como perspectiva de investigaciones futuras, no hay que olvidar que estas características que se han recogido pueden ser en un futuro ampliadas o modificadas por el avance de la tecnología, pudiendo ser relevante otro tipo de características o metodologías a seguir para el éxito en el aprendizaje de la escritura.

\section{Referencias}

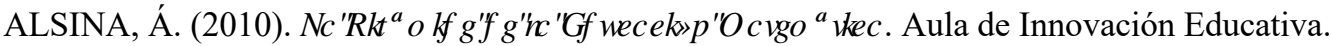

BLÁZQUEZ, F. y LUCERO, M. (2002). "Los medios y recursos en el proceso didáctico”. En Medina, A. y Salvador, F. Didáctica General (pp. 185- 218). Madrid: Pearson Educación.

CACHEIRO, M.L. (2011). Recursos educativos TIC de información, colaboración y aprendizaje. 3 L[HD \%W5 HILDG GP HARVI पHEXFDFYQ (39), 69-81.

CASADO, D., CASTRO, S. y B. GUZMÁN (2007). Las TIC en los procesos de enseñanza y aprendizaje. Laurus, Vol. 13 (23), 213-234. 
CASSANY, D. (2002). / DFRFQDCHCDHFUXW. Biblioteca para la actualización del maestro, México D.F.

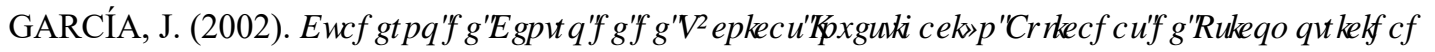
(C.I.T.A.P.) España.

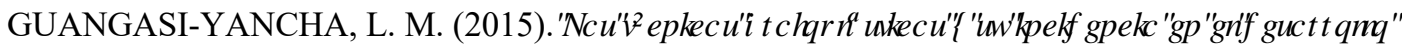
DUWUFRIHQTRVQX RVGHIRVSUP HRVDXRVGHHGXFDFY QIQIFIDO Graphoplastic techniques and their impact on artistic development in children in the first years of initial education]. Ambato: Universidad Técnica de Ambato.

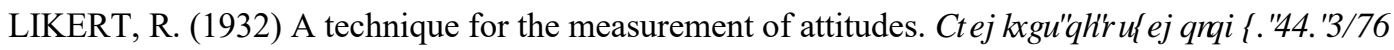
LÓPEZ, A. y LORENZO, M. (2008). La investigación educativa en el aula hospitalaria: Estudio de un caso de intervención escolar, de Glioma óptico infantil mediante videoconferencia. Píxel-Bit, Revista de Medios y Educación, 33, 29-42

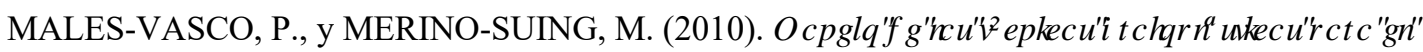

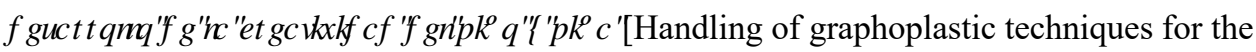
development of children's creativity]. Sangolqui: Escuela Politécnica del Ejército. Obtenido de https://n9.cl/ory5

MEDINA, A. (2009). Metodología didáctica para el desarrollo de planes de estudio en el EEES. En A. Medina, M.L. Sevillano \& De la Torre, S. (Coords.). Una universidad para el s. XXI. Espacio Europeo de Educación Superior (EEES). Una mirada transdisciplinar, ecoformadora e intercultural (pp. 195-212). Madrid: Universitas.

MONTESSORI, M. (2004). / DO HQUA\$S EVRLFQUATThe Absorbing Mind]. México: Editorial Diana

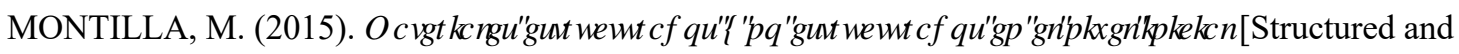
unstructured materials at entry level]. Turmero: Fedupel.

MUÑOZ RIVERA, D. (2009) La coordinación y el equilibrio en el área de Educación Física. Actividades

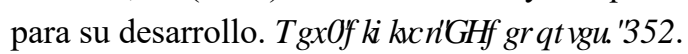

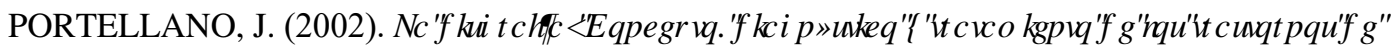

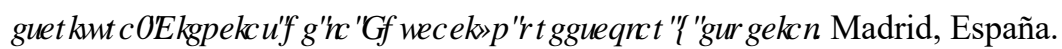

RODRÍGUEZ ABREU, M. (2010). Las bases perceptivo-motrices en primaria: la percepción espacial. HGSRUUAV, 15(146).

SUÁREZ, B. (2002). ( WWDJIDVB UFRP RURWVIV IP XW. Noriega Editores, México. D.F. 